\title{
General Ideology of Analysis Digital Medical Images in RGB Format
}

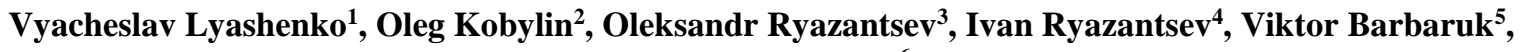 \\ Yevhen Zhychenko ${ }^{6}$ \\ ${ }^{1}$ Department of Informatics, Kharkiv National University of RadioElectronics, Ukraine, \\ lyashenko.vyacheslav@gmail.com \\ ${ }^{2}$ Department of Informatics, Kharkiv National University of RadioElectronics, Ukraine, oleg.kobylin@nure.ua \\ ${ }^{3}$ Vice-Rector for Educational and Scientific Affairs, and International Cooperation Volodymyr Dahl East \\ Ukrainian National University, Severodonetsk, Ukraine, a_ryazantsev@ukr.net \\ ${ }^{4}$ Department of Software Engineering Kharkiv National University of RadioElectronics Kharkiv, Ukraine, \\ ivan.riazantsev@nure.ua \\ ${ }^{5}$ Department of Computer Science and Engineering Volodymyr Dahl East Ukrainian National University, \\ Severodonetsk, Ukraine, vikontbvn@gmail.com \\ ${ }^{6}$ Department of Pedagogy Volodymyr Dahl East Ukrainian National University, Severodonetsk, Ukraine, \\ jychenko-ev@ukr.net
}

\begin{abstract}
Methods, technologies and various digital image processing procedures are one of the data analysis tools. At the same time, digital images can be considered as data that belong to the Big Data group. This fully applies to digital color images of cytological preparations. Thus, digital image processing techniques are one of the Big Data analysis tools. A feature of such a tool is the specificity of its use for various types of data, which are presented in the form of digital images. To do this, we examined the problematic aspects of the analysis of cytological specimens of images that are marked in the works of other authors. The paper also examined the issues of complex analysis of color images of cytological preparations. For this analysis, we used the decomposition of the original image into separate color channels, applied the ideology of wavelets to determine areas of interest and a set of morphological operations to specify such areas of interest. These methods are selected taking into account the peculiarities of images of cytological preparations. The results are shown on the example of images of cytological preparations, where megaloblastic anemia cells are also present.
\end{abstract}

Key words: Image Processing, Color Space, Wavelet Analysis, Cytological Preparations, Morphological Operations, Megaloblastic Anemia Cells

\section{INTRODUCTION}

Vision is one of the information sources. Digital images are carriers of such source of information. Digital images are a compressed representation of various images of the outside world. Digital images help to understand the processes that occur in the world around us. Various image processing technologies are used for this purpose [1]-[4]. These technologies provide additional information. Such information makes it possible to choose among various alternatives, helps to make the right decision.

However, it should be borne in mind that images of the real world can be attributed to the Big Data group. This is due to the fact that each point in the image is some information about the object. Moreover, if it is a color image, this image carries more information. Therefore, it can be argued that color images belong to the Big Data group.

RGB images of cytological preparations can be considered as one example of color images [3], [5]. RGB images of cytological preparations are very important, as they help to study a person's diseases and assess his health. This is based on the fact that we can examine the state of human health using images of cells of various internal organs. Various methods and techniques of image analysis are used for this study. We can get an initial assessment of human health. But it is also necessary to take into account the specifics of the image being investigated. Moreover, it is important to obtain additional information. Therefore, it is necessary to apply different image analysis procedures and their combinations. Harnessing a combination of different image processing techniques provides additional information. This is an important point in the study of the RGB images of cytological preparations. At the same time, the choice of tools for conducting the corresponding analysis is an important point, given the fact that images of cytological preparations belong to the Big Data group [4],[6]-[8]. 
Thus, the main goal of this study is to consider the general ideology of the analysis of cytological preparations RGB images. This approach is an important step in the diagnosis of human diseases, assessing state of his health. A common ideology also allows exploring the possibilities of obtaining additional information. This is an important point for decision making when choosing a person's treatment methods.

At the same time, the main task is to generalize some procedure for analyzing images of cytological preparations. Moreover, such a procedure should generate additional information and be based on such information.

\section{THE GENERALIZED CHARACTERISTICS OF DIGITAL IMAGE ANALYSIS PROCEDURES AND THE POSSIBILITY OF ITS USE FOR ANALYSIS OF MEDICAL IMAGES}

Various methods, technologies, and procedures are used to process digital images. Moreover, each type of image processing solves individual problems. The following tasks should be highlighted among the main [5], [9]:

improving the quality of the original image. This is necessary to visualize the source data, make decisions on visual analysis of the source image;

removal of noise and interference in the image. The solution to this problem makes it possible to remove some details in the image that prevent you from making the right decision;

highlighting areas of interest. This procedure makes it possible to reduce the area of analysis and increase the processing speed of the original image. Ultimately, this allows speeding up the decision-making process, which is important when analyzing images of cytological preparations;

identification of research objects. The solution to this problem makes it possible to automate the process of image analysis and adoption of appropriate decisions.

The use of various methods, technologies and procedures for image analysis depends on the context of the task; features of the images being investigated; the possibility of applying image processing methods. These conditions, the use of various methods, technologies and procedures for image analysis, are especially important when conducting image analysis of cytological preparations. This is due to the fact that the decisions are being made affect the state of human health. In this case, it is necessary to apply such an image processing procedure that would be suitable for the analysis of various images with the same structure of the cytological preparation.

At the same time, when processing medical images (when choosing various methods and procedures for image analysis), the specificity of such images should be taken into account.
If we are talking about images of cytological preparations (see Figure 1, as an example of such images), then the following should be considered:

images of cytological preparations are color images that are taken under a microscope. These images contain a different set of components [5], [10]. The individual constituent parts of the original image may have a very small size. Therefore, one should apply carefully the procedures to improve the quality of the original image or remove interference, noise. Such processing of images of cytological preparations should be carried out taking into account preliminary identification of the size for the area of interest;

the presence of staining of cytological preparations. This allows you to highlight some areas of interest. But, at the same time, we may lose some details due to the imposition of color and the features of its manifestation for different cytological preparations [5]. For example, Figure 1 presents images of the same structure of the cytological preparation, but these images have different color characteristics;

images of cytological preparations have a complex structure (see Figure 1). This is due to the fact that the image of cytological preparations may contain various elements [10]. Therefore, it is important to know which particular element needs to be identified. For example, in Figure 1 megaloblastic anemia cells are the main structure of the cytological preparation. Therefore, further we will consider the procedure for isolation of megaloblastic anemia cells.

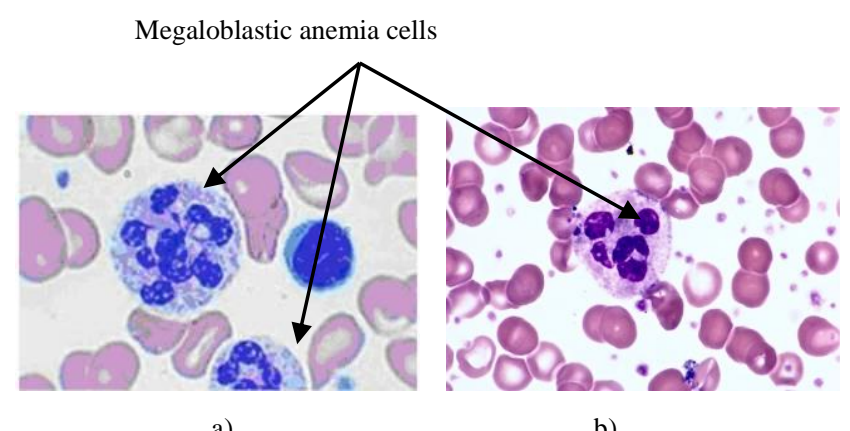

a)

b)

Figure 1: Example digital medical images

As an example of using of various methods, technologies and procedures for analyzing images of cytological preparations, we cite a number of works by some authors.

O. A. Artyukhova and A. V. Samorodov consider various procedures to improve the quality of imaging of cytological preparations and their automated processing [11]. It is shown in the paper that Gausovsky filters and filters based on the Bayesian spectrum are most suitable for this procedure. At the same time, it was noted that the contrast and sharpness of the 
Vyacheslav Lyashenko et al., International Journal of Emerging Trends in Engineering Research, 8(5), May 2020, 1647 - 1655

original image depends on the size of the individual structural components of cytological preparations.

The possibilities of using wavelets ideology when analyzing cytological preparations images were studied in [10]. The authors note the advisability of using wavelets to highlight various components of cytological preparations. For this, it is possible to make consistent the sizes of individual components of cytological preparations and wavelet parameters. This suggests the preference for using the ideology of wavelets in the processing of images of cytological preparations. The authors of the work also note the need for decomposition of color images into separate color channels to obtain more complete information about the images being studied.

K. Chain, T. Legesse, J. E. Heath and P. N. Staats consider the use of digital medical image analysis methods to improve the accuracy of diagnosis of human diseases [12]. In this case, special attention is paid to errors that arise due to the use of various methods of coloring the corresponding digital images. It is indicated that to increase the accuracy of diagnosis, it is necessary to use modified staining methods. The importance of obtaining additional information to eliminate errors in staining of cytological preparations is also noted.

In the work, edited by M.M. Bui and L. Pantanowitz, various methods for studying images of cytological preparations were analyzed [13]. At the same time, if was noted in the paper that most of these methods for the analyzing digital images should be adapted to solve a specific problem or use a certain sequence of digital methods of image processing to obtain results. Among the most used methods for analyzing digital images, the authors indicate various filtering methods, segmentation methods to highlight areas of interest. Similar studies can also be found in [14]-[16].

The paper [17] gives practical results of using digital processing methods in the study of images of cytological preparations. The authors note the importance of using digital processing of the obtained images for the diagnosis of various diseases. Among these methods, various filters are distinguished for suppressing noise and improving the quality of the original image, various methods for highlighting areas of interest. At the same time, the question remains open about the automatic use of digital processing methods in the analysis of images of cytological preparations.

Thus, some authors use various methods of analyzing digital images to study images of cytological preparations. This expands the possibilities of using digital processing methods for the analysis of images of cytological preparations. Nevertheless, the important issue is the use of a certain combination of methods and approaches to solve the tasks and achieve the result. Therefore, many authors believe that special attention should be paid to obtaining additional information and its use to solve the tasks.

\section{KEY TOOLS FOR THE GENERAL IDEOLOGY OF MEDICAL IMAGE ANALYSIS IN RGB FORMAT}

When considering the general ideology of images of cytological preparations analysis, we take into account the features of such images, as well as the main goal of this study. Then we need to: get additional information; take into account the complex structure of the cytological preparation and the presence of objects of various sizes in this structure.

Note that one of the most common ways of representing color images of cytological preparations is to present them in RGB format. An image in the RGB color system consists of three color channels - R (red), G (green) and B (blue) [18]. Unlike other color formats for representing digital images, each channel of the RGB format can be considered equal. At the same time, each color channel allows you to consider a specific frequency region of the image. Thus, we get three sources of additional information. In addition, each source of additional information allows you to take into account all the features of the image.

Let we have the original image $-\mathrm{Q}(\mathrm{i}, \mathrm{j})=\mathrm{q}$, where $\mathrm{Q}$ is the input image of cytological preparations size $\mathrm{M} \times \mathrm{N},(\mathrm{i}, \mathrm{j})$ are the current coordinates of the points of the original image $\mathrm{Q}$ $(i=\overline{1, M}, j=\overline{1, N}), g$ is the brightness of the image at a point $Q(i, j)$.

With this consideration for each source image $\mathrm{Q}$, we have a set of three images $(\mathrm{Q}(\mathrm{i}, \mathrm{j})=\mathrm{QR}(\mathrm{i}, \mathrm{j}) \oplus \mathrm{QG}(\mathrm{i}, \mathrm{j}) \oplus \mathrm{QB}(\mathrm{i}, \mathrm{j}))$ :

$\mathrm{QR}(\mathrm{i}, \mathrm{j})=\mathrm{rq}$ - original image in color channel $\mathrm{R}$, where rq is the brightness value of the image at the point $\mathrm{QR}(\mathrm{i}, \mathrm{j})$;

$\mathrm{QG}(\mathrm{i}, \mathrm{j})=\mathrm{gq}$ - original image in color channel $\mathrm{G}$, where $\mathrm{gq}$ is the brightness value of the image at the point $\mathrm{QG}(\mathrm{i}, \mathrm{j})$;

$\mathrm{QB}(\mathrm{i}, \mathrm{j})=\mathrm{bq}$ - original image in color channel $\mathrm{B}$, where $\mathrm{bq}$ is the brightness value of the image at the point $\mathrm{QB}(\mathrm{i}, \mathrm{j})$;

$\oplus$ - operation combining the individual component color images in a single image.

Thus, we have prepared the basis for more information. Such information can be obtained by a detailed review of the structure of the original image.

We can also compare our sources with each other. Such a comparison takes into account the fact that, as a rule, color images are transformed into monochrome images for their subsequent processing. Then we can compare the source color 
image, which is transformed into a monochrome image with images for each color channel. For this we use concepts:

entropy ( ENT ). Entropy is a statistical measure of randomness that can be used to characterize the texture of the image. Then we can say how much the images we are exploring are very different. In this case, this is a qualitative assessment in comparing images;

sum of the absolute differences of the brightness values of each point of two images (SUM). Then we can say how different the images we are exploring are. In this case, this is a quantitative assessment in the comparison of images.

Next, we need to take into account the features of images of cytological preparations, the presence of objects of various sizes, taking into account the need to identify megaloblastic anemia cells. To solve this problem, we will use wavelet analysis [10]. The choice of this approach is based on the fact that we need to identify objects of small sizes. Such identification can be done by wavelet analysis based on tuning wavelets to search for the corresponding features of cytological preparations.

The wavelet methodology is based on the search for differences in brightness levels in the original image. Such differences can we find if we analyze some of the original image points $Q(i, j)$ in its vicinity $\varepsilon$ for each component color images ( $Q R(i, j), Q G(i, j), Q B(i, j))$. This analysis is based on the analysis of changes in brightness $q$ at different points in the image.

At the same time, one can choose such a wavelet that allows identifying swings in the best way from the point of view of image analysis of cytological preparations. Thus, wavelet analysis allows one to variably approach the solution of specific problems of analyzing images of cytological preparations.

To search for such changes, we examine each component of the image: row by row, column by column. Using wavelets, we determine the differences in brightness levels separately for each image line and separately for each image column [10]. This approach allows you to take into account all possible differences in brightness levels of the image that is being studied. The images obtained in this way are basic for subsequent analysis. As a result, we get three images (for each color channel), which were analyzed using the wavelet ideology. Then we have the basis for further analysis and clarification of areas of interest. For the purpose of clarifying areas of interest, morphological operations can be used as a tool for relevant analysis. Morphological operations allow us to clarify the identification of objects that we are exploring. Therefore, among such operations, it should be distinguished (based on the fulfillment of certain logical conditions): operations of combining image points, deleting image points, adding image points. Moreover, having several sources of information about areas of interest, you can combine the output procedure. This can be done on the basis of semantic information about the object under study, as well as using information about objects within the individual color channels.

The generalized scheme for the analysis of RGB images of cytological preparations consists of three stages and is presented in Figure 2.

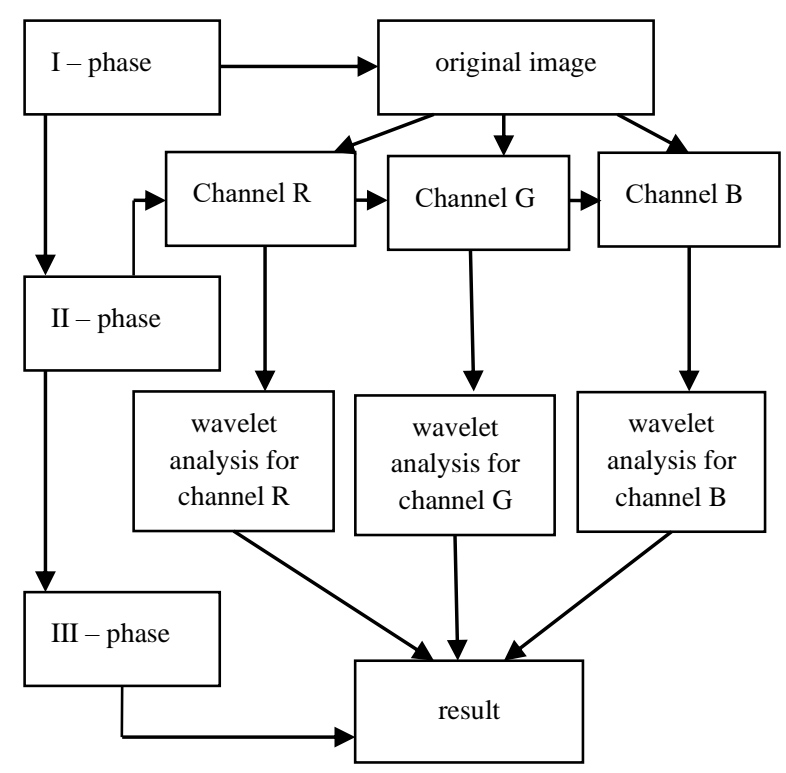

Figure 2: Generalized scheme for the analysis of RGB images of cytological preparations

The essence of each of the stages of the generalized scheme for the analysis of RGB images of cytological preparations is as follows:

stage 1 - we perform the decomposition of the original image into individual color components;

stage 2 - apply wavelet analysis for each color component of the original image of cytological preparations;

stage 3 - we use tools of morphological analysis (individual morphological operations) to solve the specific problem of processing images of cytological preparations.

\section{RESULTS OF EXPERIMENTAL STUDIES}

Let us consider the implementation of a generalized scheme for analyzing RGB images of cytological preparations using specific examples. To do this, consider the data in Figure 1. 
First of all, we consider the 1st stage of the generalized scheme - namely, the decomposition of the original image into separate color channels. We also show the feasibility of carrying out such a stage of the general scheme. Here, by expediency we mean the possibility of obtaining additional information without a significant change in the primary information. To do this, we consider monochrome images for the source images and their decomposition into separate color channels. For comparison, we will use the indicators ENT and SUM (see above). In Figure 3 shows monochrome images for the original color images of Figure 1.

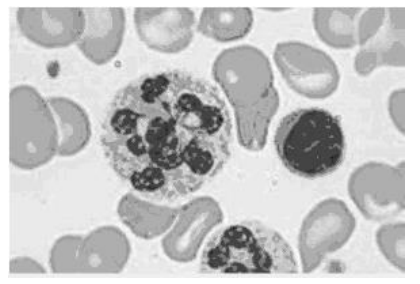

a)

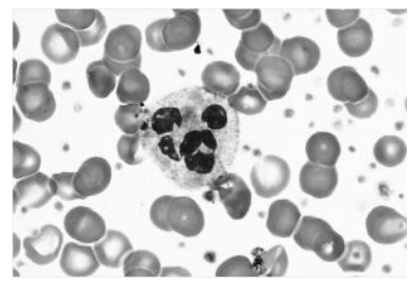

b)
Figure 3: Monochrome images for the original color images of Figure 1.

Figure 4 shows the decomposition of the original image (Figure 1a). These images are presented as black and white images (color channel $\mathrm{R}-$ Figure $4 \mathrm{a}, \mathrm{G}-$ Figure $4 \mathrm{~b}, \mathrm{~B}-$ Figure 4c).

Figure 5 shows the decomposition of the original image (Figure $1 \mathrm{~b}$ ). These images are presented as black and white images (color channel $\mathrm{R}$ - Figure 5a, G - Figure 5b, B Figure 5c).

We can see that the images for the individual color channels are different from each other. The largest difference is characteristic of color channel B. Such a difference can be seen in different saturations of black and white images. This is due to the method of coloring the cytological preparation. You can also see that the monochrome image (Figure 3) and the images of the individual color channels coincide in many ways (Figure 4 and Figure 5).

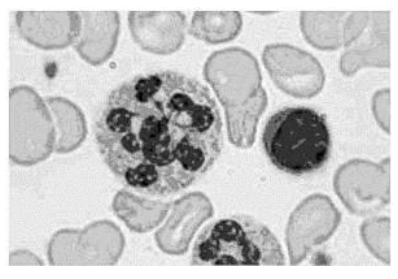

a)

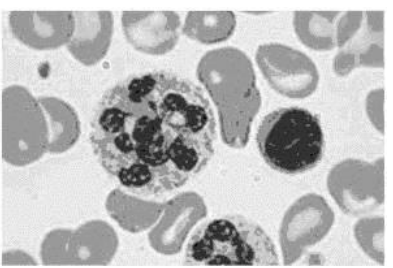

b)

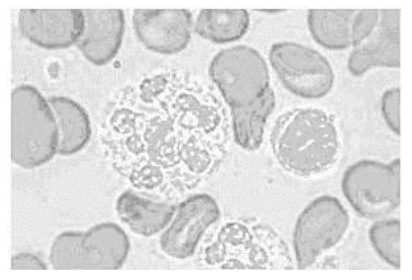

c)

Figure 4: Decomposition of the original image (Figure 1a) in separate color channels [19]

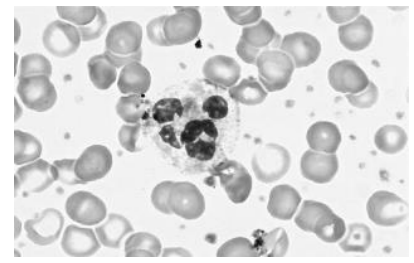

a)

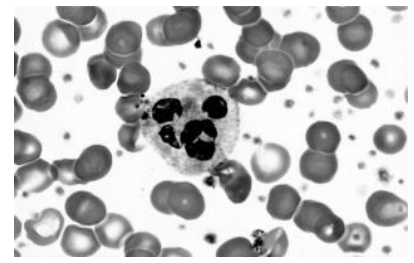

b)

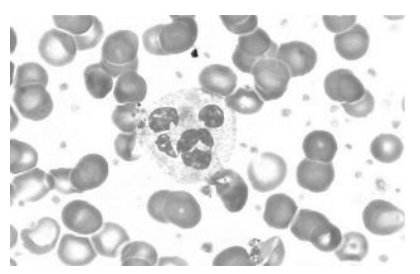

c)

Figure 5: Decomposition of the original image (Figure 1в) in separate color channels [19]

Moreover, the characteristic texture of the image of the combination of various components of cytological preparations remains the same. In particular, the calculation of the values ENT shows minor changes in this indicator within the same image structures of cytological preparations (Table $1)$.

Table 1: The value of the indicator ENT for various images of cytological preparations

\begin{tabular}{|l|l|l|l|l|}
\hline Image & $\begin{array}{l}\text { All } \\
\text { images } \\
\text { (monochr } \\
\text { ome) }\end{array}$ & Channel R & Channel G & Channel B \\
\hline Figure 1a & 6.3092 & 6.7021 & 6.4622 & 6.1204 \\
\hline Figure 1b & 6.1403 & 6.0624 & 6.4306 & 5.3040 \\
\hline
\end{tabular}

Table 2 shows the indicator values SUM for the monochrome image (M) and individual channels of the color image in Figure 1a.

Table 2: The values of the indicator SUM for a monochrome image and individual channels of a color image Figure 1a

\begin{tabular}{|l|l|l|l|l|}
\hline & M & R & G & B \\
\hline M & 0 & 576457 & 389537 & 1495430 \\
\hline R & 576457 & 0 & 791942 & 1404215 \\
\hline G & 389537 & 791942 & 0 & 1854977 \\
\hline B & 1495430 & 1404215 & 1854977 & 0 \\
\hline
\end{tabular}


Table 3 shows the indicator values SUM for the monochrome image (M) and individual channels of the color image in Figure 1b.

Table 3: The values of the indicator SUM for a monochrome image and individual channels of a color image Figure 1b

\begin{tabular}{|l|l|l|l|l|}
\hline & M & R & G & B \\
\hline M & 0 & 13528294 & 8789660 & 12034421 \\
\hline R & 13528294 & 0 & 22304646 & 8383463 \\
\hline G & 8789660 & 22304646 & 0 & 19633697 \\
\hline B & 12034421 & 8383463 & 19633697 & 0 \\
\hline
\end{tabular}

The data values of Table 2 and Table 3 characterize a quantitative measure of the difference in images with each other.

Thus, we obtained various sources of information for identifying megaloblastic anemia cells. This is the basis for using wavelet analysis.

Figure 6 shows the results of the wavelet analysis for the images in Figure 4. Figure 7 shows the results of the wavelet analysis for the images in Figure 5.

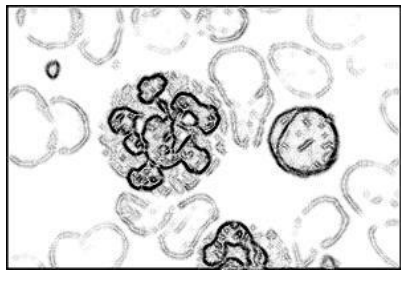

a)

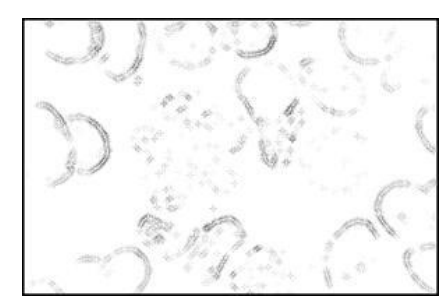

c)

Figure 6: Results of the wavelet analysis for images in Figure 4 [19]

For the wavelet analysis, we used the Daubechies wavelet $(\mathrm{db} 1)$. This wavelet allows you to best synchronize the selection of areas of interest of various sizes [10].

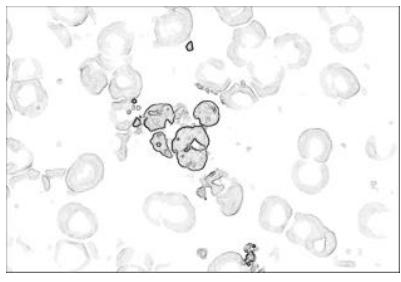

a)

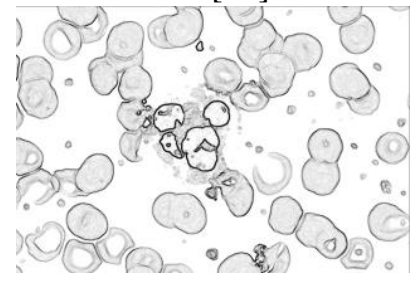

b)

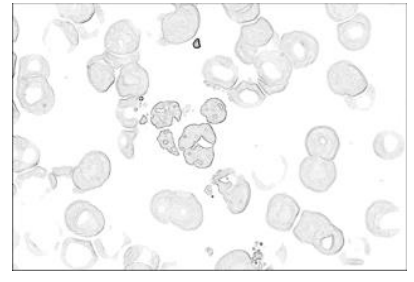

c)

Figure 7: Results of the wavelet analysis for images in Figure 5 [19]

We see that the results of the wavelet analysis are also different for each component of the color channels. Such a difference is manifested in a different allocation of areas of interest on images of cytological preparations. But we got more information. This allows for a more accurate analysis. We can also choose different strategies depending on which areas of interest are of interest. In order to show the differences between the results of the wavelet analysis, we used Pareto histograms.

In particular, in Figure 8 shows the Pareto histograms for the data in Figure 7 (respectively). These data reflect the various degrees of prevalence of the brightness characteristics of the images after wavelet processing.

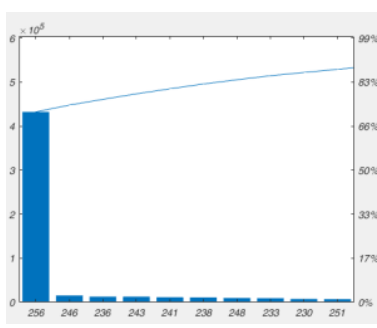

a)

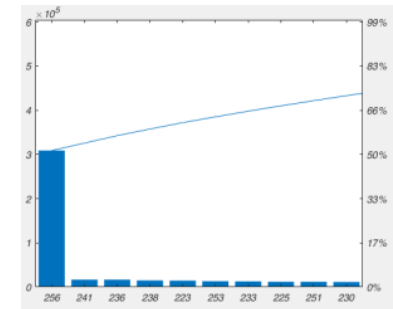

b)

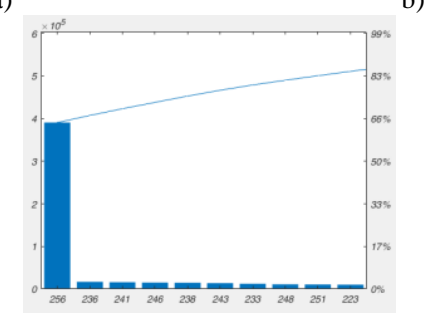

c)

Figure 8: Pareto histograms for data Figure 7

Pareto histograms also provide a quantitative interpretation for the semantic basis of morphological analysis.

For example, data analysis in Figure 6 and Figure 7 show that the results of the wavelet analysis for color channel B are significantly different from the results for color channel $\mathrm{R}$ and G. This fact can be used to carry out the corresponding morphological analysis (identification of megaloblastic anemia cells). 
In particular, to solve the problem of identifying the identification of megaloblastic anemia cells, it is advisable to select the results of wavelet processing for the color channel $\mathrm{B}$ as the main (structuring) element. This choice allows you to remove elements of the cellular structure of the cytological preparation for channels $\mathrm{R}$ and $\mathrm{G}$ that are presented for the channel $\mathrm{B}$. We do the deletion taking into account the values of the Pareto histogram. This allows you to save the elements of cellular structures with the highest intensities for the color channels $\mathrm{R}$ and $\mathrm{G}$ in comparison with the channel $\mathrm{B}$. In this case, we adhere to the strategy of maximizing the results that are most manifested for the color channels $R$ and $G$. In particular, in automatic mode, these channels can be selected based on the data table 1 (maximum value of the indicator ENT ).

Figure 9 shows the results of removing the image points in Figure 6a, Figure 6b and Figure 7a, Figure 7b, respectively. We can see that such a deletion does not distort (significantly) the identification of megaloblastic anemia cells.

But we also see elements of the cellular structure that are not megaloblastic anemia cells. Therefore, we make the association of individual images. This can be done using the operation of morphological intersection.

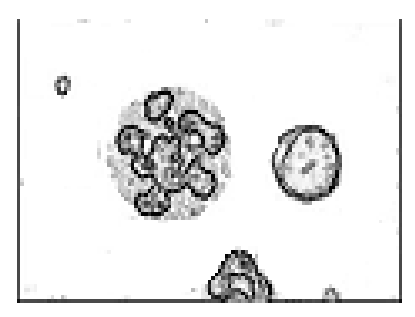

a)

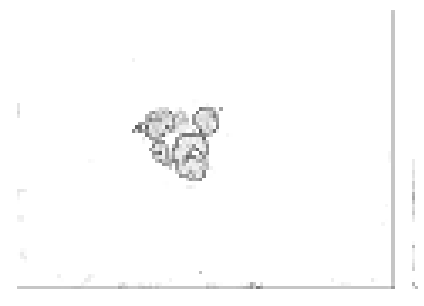

c)

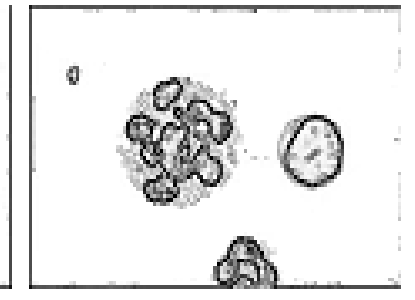

b)

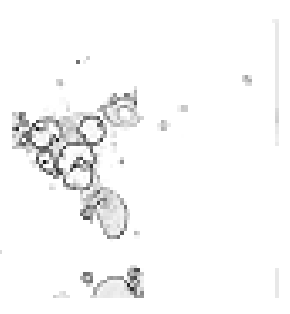

d)
Figure 9: Result of morphological removal of objects

Figure 10 shows the results of the morphological intersection for the identification of megaloblastic anemia cells.

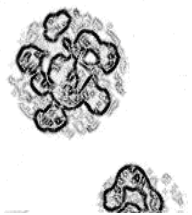

a)

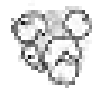

b)
Figure 10: Result of morphological intersection

The morphological intersection is the result of the intersection of the set of points present in Figure 9a, Figure 9b, Figure 6c (this is Figure 10a) and Figure 9c, Figure 9d, Figure 7c (this is Figure 10b), respectively.

To identify other objects in the image of cytological preparations or guided by a different strategy for selecting the studied objects, we can specify a different morphology. Such an approach allows honoring those shortcomings that various authors have noted when considering various aspects of image processing of cytological preparations. So, for example, considering individual color channels and various strategies for choosing and using morphological operations, we can reduce the errors in identifying the area of interest that arise as a result of using various techniques for staining cytological preparations. At the same time, the use of wavelet analysis allows one to minimize the loss of small objects in the general structure of the components of cytological preparations.

If we talk about the probabilistic characteristics of the considered approach, it should be noted the complexity of this issue. In this aspect, we relied more on increasing the degree of visualization of the data obtained in the final result. At the same time, the validity of the application of certain approaches and the expediency of their use for the analysis of images of cytological preparations are based on such quantitative characteristics as: entropy, the sum of the absolute differences in the brightness values of the studied images, and the Pareto histogram.

\section{CONCLUSION}

The use of digital image processing methods is widespread for visualization and analysis of medical data. At the same time, it is the field of application of such data processing methods that makes it difficult to solve the problems posed. Since in this case the need for a qualitative and quick analysis of the state of human health. Therefore, the analysis of biomedical data is a complex problem.

Therefore, in order to solve various problems that concern, in particular, image processing of cytological preparations, additional information is needed. For more information, we used the technique of decomposing images into color components, as well as the wavelet ideology to search for areas of interest. To summarize the information and identify objects of interest, we used morphological analysis. At the same time, we showed the possibility of choosing various 
strategies for morphological analysis. The basis for choosing such strategies is wavelet analysis data for various color components of the original image.

The possibility of using the procedures and approaches for analyzing digital images considered in this work is based on the features of the analysis of images of cytological preparations. The results of this study can be used to develop automatic procedures for analyzing images of cytological preparations.

\section{REFERENCES}

1. M. Saha, R. Mukherjee, and C. Chakraborty. Computer-aided diagnosis of breast cancer using cytological images: A systematic review, Tissue and Cell, Vol. 48, no. 5, pp. 461-474, 2016. https://doi.org/10.1016/j.tice.2016.07.006

2. A. Capitanio, R. E. Dina, and D. Treanor. Digital cytology: A short review of technical and methodological approaches and applications, Cytopathology, Vol. 29, no. 4, pp. 317-325, 2018. https://doi.org/10.1111/cyt.12554

3. V. Lyashenko, M. A. Babker, and V. A. Lyubchenko. Wavelet Analysis of Cytological Preparations Image in Different Color Systems, Open Access Library Journal, Vol. 4, pp. e3760, 2017. https://doi.org/10.4236/oalib.1103760

4. A. M. Babker, A. E. A. Altoum, I. Tvoroshenko, and V. Lyashenko. Information technologies of the processing of the spaces of the states of a complex biophysical object in the intellectual medical system health, International Journal of Advanced Trends in Computer Science and Engineering, Vol. 8, no 6, pp. 3221-3227. https://doi.org/10.30534/ijatcse/2019/89862019

5. N. Dey, A. S. Ashour, A. S. Ashour, and A. Singh. Digital analysis of microscopic images in medicine, Journal of Advanced Microscopy Research, Vol. 10, no. 1, pp. 1-13, 2015.

https://doi.org/10.1166/jamr.2015.1229

6. M. Ayaz, I. Tvoroshenko, J. H. Baker, and V. Lyashenko. Modeling the Structure of Intellectual Means of Decision-Making Using a System-Oriented NFO Approach, International Journal of Emerging Trends in Engineering Research, Vol. 7, no. 11, pp. 460-465, 2019. https://doi.org/10.30534/ijeter/2019/107112019

7. M. Ayaz, I. Tvoroshenko, J. H. Baker, and V. Lyashenko. Computational Complexity of the Accessory Function Setting Mechanism in Fuzzy Intellectual Systems, International Journal of Advanced Trends in Computer Science and Engineering, Vol. 8, no. 5, pp. 2370-2377, 2019.

https://doi.org/10.30534/ijatcse/2019/ 77852019

8. P. Orobinskyi, and V. Lyashenko. The Task of Identifying the Various Objects' Boundary Points of the Medical Image as the Optimal Path Search on the Graph, International Journal of Emerging Trends in
Engineering Research, Vol. 8, no. 3, pp. 824-829, 2020. https://doi.org/10.30534/ijeter/2020/36832020

9. V. Lyashenko, O. Kobylin, and Y. Baranchykov. Ideology of Image Processing in Infocommunication Systems, In 2018 International Scientific-Practical Conference Problems of Infocommunications. Science and Technology (PIC S\&T), pp. 47-50, 2018. https://doi.org/10.1109/INFOCOMMST.2018.8632124

10. V. Lyashenko, A. Babker, and O. Kobylin. The methodology of wavelet analysis as a tool for cytology preparations image processing, Cukurova Medical Journal, Vol. 41, no. 3, pp. 453-463, 2016. https://doi.org/10.17826/cukmedj.237468

11. O. A. Artyukhova, and A. V. Samorodov. Investigation of image sharpness characteristics in the field of automated microscopy of cytological preparations, Pattern Recognition and Image Analysis, Vol. 21, no. 2, pp. 171-175, 2011. https://doi.org/10.1134/S1054661811020118

12. K. Chain, T. Legesse, J. E. Heath, and P. N. Staats. Digital image-assisted quantitative nuclear analysis improves diagnostic accuracy of thyroid fine-needle aspiration cytology, Cancer cytopathology, Vol. 127, no. 8, pp. 501-513, 2019. https://doi.org/10.1002/cncy.22120

13. M. M. Bui, and L. Pantanowitz (eds). Modern Techniques in Cytopathology. Monogr Clin Cytol. Basel, Karger, Vol. 25, pp. 91-98, 2020. https://doi.org/10.1159/000455776

14. D. Dov, S. Kovalsky, J. Cohen, D. Range, R. Henao, and L. Carin. Thyroid cancer malignancy prediction from whole slide cytopathology images, arXiv preprint, 2019, arXiv:1904.00839.

15. L. Quaroni, T. Zlateva, K. Wehbe, and G. Cinque. Infrared imaging of small molecules in living cells: from in vitro metabolic analysis to cytopathology, Faraday discussions, Vol. 187, pp. 259-271, 2016. https://doi.org/10.1039/C5FD00156K

16. E. B. Kablan, H. Dogan, M. Ekinci, M. E. Ercin, and S. Ersoz. Stain independent nuclei segmentation of cytopathology images: A case study in pleural effusion, In 2019 Medical Technologies Congress (TIPTEKNO), pp. 1-4, 2019. https://doi.org/10.1109/TIPTEKNO.2019.8895174

17. I. A. Kruglova, S. V. Zinoviev, O. V. Utkin, A. N. Denisenko, O. E. Ilyinskaya, and M. A. Moskvichev. Digital image in the practice of Cytology: a pilot study, Klinicheskaia laboratornaia diagnostika, Vol. 64, no. 11, pp. 649-653, 2019. https://doi.org/10.18821/0869-2084-2019-64-11-649-65 3

18. F. Fereidouni, C. Griffin, A. Todd, and R. Levenson. Multispectral analysis tools can increase utility of RGB color images in histology, Journal of Optics, Vol. 20, no. 4, pp. 044007, 2018.

19. V. Lyashenko, O. Kobylin, O. Ryazantsev, I. Ryazantsev, V. Barbaruk, and Y. Zhychenko, Wavelet Ideology and Morphological Operations for Analyzing RGB Images of Cytological Preparations, In 7 th International Conference on Future Internet of Things 
Vyacheslav Lyashenko et al., International Journal of Emerging Trends in Engineering Research, 8(5), May 2020, 1647 - 1655

and Cloud Workshops (FiCloudW), Istanbul, Turkey, pp. 119-122, 2019.

https://doi.org/10.1109/FiCloudW.2019.00034 